\title{
Reconstruction of Atrial Ectopic Focal and Re-entrant Excitations from Body Surface Potentials. Insights from 3D Virtual Human Atria and Torso
}

\author{
Erick A Perez Alday, Michael A Colman, Henggui Zhang \\ University of Manchester, Manchester, United Kingdom
}

\begin{abstract}
Non-invasive electrocardiographic imaging has been seen as a painless and economic method to map the electrical functions of the heart. However, it is still a great challenge to obtain accurate reconstruction of cardiac electrical activity from body surface potentials (BSP) due to the ill-posed behaviour of the cardiac inverse-problem. Though some advances have been made in solving the inverse-problem, few studies have been conducted for the atria, which have dramatic differences to the ventricles in their anatomical structures and electrophysiological properties. It is unclear either how the spatial resolution of electrodes on the BSP and rapid excitation rates of atrial activation during atrial fibrillation affect the accuracy of the inverse-problem.

In this study, we used a biophysically detailed model of the human atria and torso to investigate effects of multilead ECG on the accuracy of reconstructed atrial excitation pattern on the epicardiac surface during the time courses of atrial fibrillation induced by electrical remodelling. It was shown that the solution of the atrial inverse-problem was dependent on the spatial resolution of electrodes on the body surface. The solution was also influenced by the morphology of the AP, rate and types of atrial excitation as well as the implantation of variant orders of the Tikhonov regularization method.
\end{abstract}

\section{Introduction}

The electrocardiographic imaging, based on the cardiac inverse problem solution, provides a promising method for non-invasive diagnosis and locating the origins of cardiac arrhythmias (1). However, due to the ill-posedness of the problem, prior empiric information is required to constrain the problem in order to achieve a reliable solution (1). Due to the limited number of electrodes (i.e., the spatial resolution of the measured signals) on the body surface and unavoidable noise of the measured signals, it is still a challenge to obtain a reliable and accurate solution of the cardiac inverse problem.
Previous studies on the ventricular inverse-problem have shown that the number of electrodes on the body surface has dramatic effects on the solution $(2,3)$. As few studies have been conducted to investigate the atrial inverse-problem $(4,5)$, it is unclear if the findings in ventricular inverse-problem are applicable to the atriainverse problem given the dramatic differences in their electrophysiology and anatomical structures.

Therefore, there is a pressing need to develop noninvasive methods to improve the diagnosis of the atrial fibrillation (AF) origins. AF is one of the most common cardiac arrhythmia associated with a high risk of morbidity and mortality (6). Especially AF has a high incidence ( $>2 \%$ of population with ageing over 65 ), and such an incidence is expected to be increased due to the ageing society in the next decade (7). In addition, the morphology of the atrial action potential (AP) varies from almost a triangular to an almost squared shapes from region to region (8), which differs to the ventricular epicardial APs. As AF is normally associated with either rapid focal pacing (spontaneous rapid firing of nonpacemaker cells) (9), fibrillatory conduction of multiple wavelets (10), re-entrant excitation scroll waves (i.e., rotors) $(9,10)$ or combined of the three, its electrical activity is characterised by rapid and irregular electrical activation of the atria. All of these impose a challenge to a reliable solution to the atrial inverse-problem.

Implementation of variant orders of the Tikhonov regularization method may also affect the solution of the cardiac inverse-problem. However, it is still unclear how this would affect optimal solutions for cardiac excitation waves with different rates and dynamical behaviors of excitation waves during AF.

The aim of this study was to use a biophysically detailed model of the human atria and torso to investigate the effects of variant spatial resolution on the BSP and three different orders of the Tikhonov regularization method on reconstructed atrial surface potentials during atrial excitation associated with either focal activity or reentrant excitation waves. Effects of the intrinsic electrophysiological heterogeneity of the atria and fibrillationinduced electrical remodelling were considered. 


\section{Methods}

\subsection{Atrial-torso model}

A previously validated biophysically detailed computational model of the three-dimensional (3D) human atria and torso $(11,12)$ was used to simulate ectopic focal and re-entrant atrial excitation conditions (Figure1A). Details of the atrial-torso model (Figure1C), which accounts for major anatomical structures (Figure 1 D), intrinsic differences in the electrophysiological heterogeneity (13) and variant electrical conductivities of different types of internal tissues within the body (Figure1C) (11), have been considered in the model (11). The atria-torso model has been validated and used to develop an algorithm to diagnose atrial ectopic origin from multi-lead ECG by using the forward problem solutions (Figure 1E) in our previous study (11). Details of the atrial cell models and 3D simulation protocols can be found in Colman et al (8); details of the atria-torso model development, validation and simulation protocols can be found in Perez Alday et al. (11).

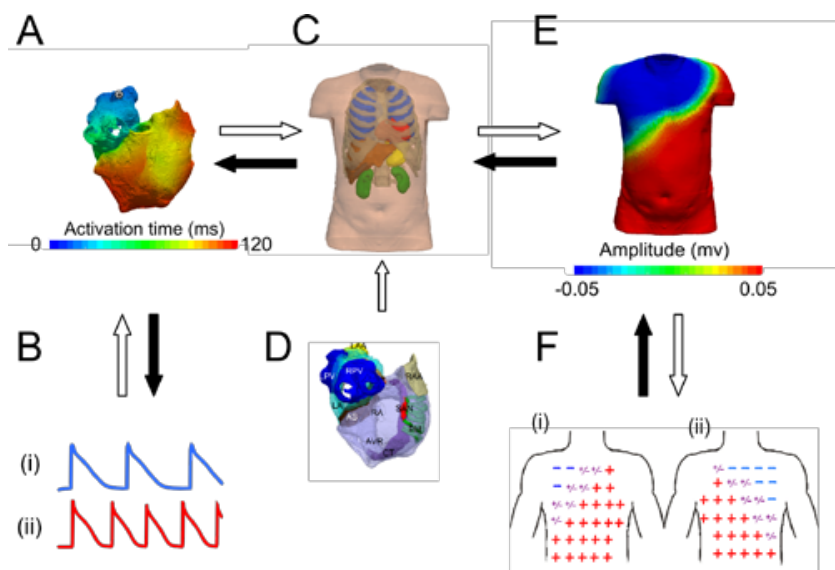

Figure 1. Schematic illustration of solving the forward (open arrow) and inverse (solid arrow) atrial problem pathways. (A) Atria activation pattern during an ectopic activity; (B) Recorded epicardial potential in slow (i) and fast (ii) rates; (C) the integrated torso model; (D) the atria model; (E) simulated BSP; (F) body surface polarity pattern reconstructed from a multi-lead ECG system.

\subsection{Simulating atrial ectopic foci and re-entry}

Atrial ectopic foci and re-entry were initiated in different regions of the atria (Figure 2Ai-ii) $(8,14)$. Parameters of the Colman et al. model of single human atrial myocytes were modified to incorporate experimentally observed AF-induced electrical remodelling of ion channels (8), which resulted in an abbreviated AP duration (APD) (Figure 2B). Such ion channel remodelling altered the morphology of atrial AP, which changed from a squared (Figure 2Bi-RAA) to a triangular shape (Figure 2Bii-RAA).

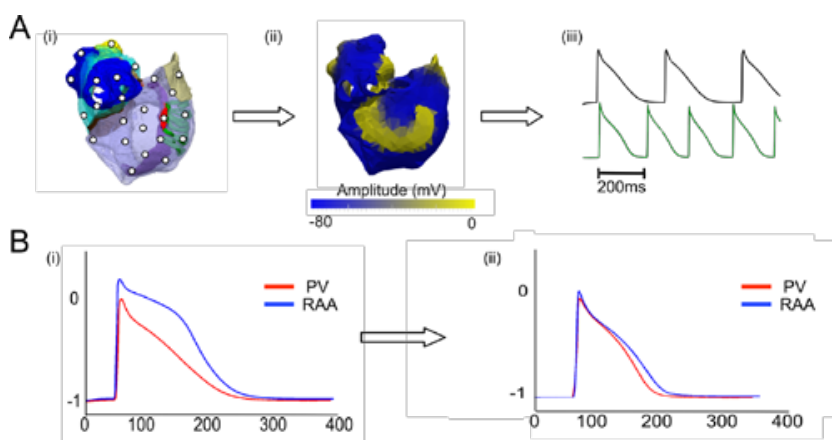

Figure 2. Illustration of the initiation of atrial ectopic and re-entrant excitation activities. (A) Positions of ectopic and re-entrant origins on the atria (i); re-entry activation with its tip located in the sino-atrial node (SAN) (ii); recorded local APs with different rates (iii). (B) morphology of APs for different types of atrial cells located in the pulmonary veins (PV-red line) and right atria appendage (blue line- RAA) for control (i) and AFremodelling conditions.

\subsection{Inverse reconstruction of the atrial surface electrical excitation}

Based on the computed BSP from the atria-torso model (11), epicardial excitation patterns of the atria were reconstructed. The transfer matrix, which relates the BSP with the potential on the atrial surface, was calculated using Green's Theorem and a boundary element discretization (15). Zero, first and second order Tikhonov regularization were implemented to find the potentials on the surface of the atria (16). The triangle method was used to automatically find the best regularization parameter in each L-curve (17). The inverse solution was validated by comparing the reconstructed atria surface excitation pattern with the "real" (i.e., simulated atrial excitation pattern) with the same spatial resolution.

Atrial epicardial excitation patterns were reconstructed at different timings using 64, 256 and 512 electrodes, which were compared to the actual activation pattern produced by the atria model. A linear interpolation was used in each case.

In order to compare the effects of different Tikhonov regularization methods and lead systems on the inverse problem solution, whole atrial time activation maps were computed. Real and reconstructed epicardial potentials from different points on the epicardial surface were also obtained (the positions were selected at different sites with different electrophysiology (Figure 2B)). From those epicardial potential, relative errors (RE) as a function of time were calculated to quantify the specific differences. REs are the difference between real and solution data divided by the magnitude of the real value. 


\section{Results}

\subsection{Reconstructed atrial surface excitation in ectopic focus}

In order to investigate the effects of different AP morphologies on the reconstruction accuracy, REs were obtained using the atrial model with three different electrophysiological conditions. Results are presented in Figure 3 for homogeneous atria with a square AP morphology (Figure 3B); homogeneous atria with a triangular and shortened AP morphology (as is observed in chronic AF remodelling - Figure 3C); heterogeneous with both square (Figure 3D-i) and triangular (Figure 3Dii) morphologies from two sites of the atria, one on the right atrial appendage (Figure 3Ai) and the other on the left atria (Figure 3Aii).

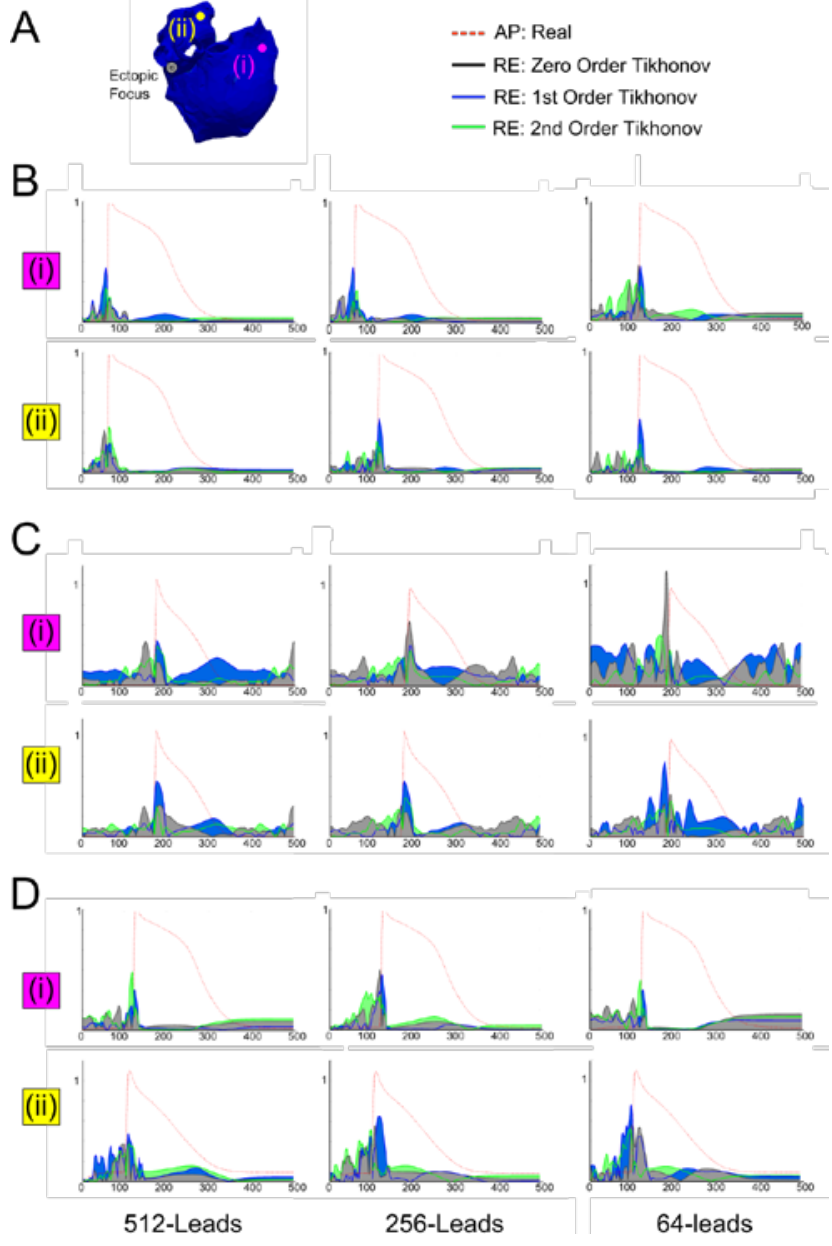

Figure 3. Relative error (RE) of epi-cardial reconstruction of an ectopic atrial activity with its origin located in the pulmonary veins region using 512-, 256- and 64-lead ECG. The real potential (red line) is plotted along with the RE using zero (black line), first (blue line) and second (green line) order Tikhonov regularization method. The reconstructions were obtained for homogeneous squared
(B), homogenous remodelling triangular (C) and heterogeneous (D) cases. The ectopic focus can be observed in A (black and white spot). Left (i) and right atria appendage (ii) epicardial potentials are observed in each case.

It was shown that when AP was changed from a squared to a triangular morphology, there was a significant increase in RE (Figure $3 \mathrm{~B}$ and $3 \mathrm{C}$ ). In the case with a heterogeneous atria (Figure 3D-i and D-ii), the computed RE was greater than the one with a homogeneously squared AP, but smaller than the one with a homogeneously triangular AP.

The use of 512-lead ECG system presented the lower RE signals, with a close solution obtained from the 256lead system in each case. However, the 64-lead ECG showed lower RE signals in the case of squared and homogeneous electrophysiology. In addition, the first order Tikhonov regularization showed the larger RE signals in most of the cases (Figure 3 blue line). For homogeneous APs, second and first order showed similar results. However, a smaller RE signal was seen in the second order case with square APs (Figure 3B), and in zero order for triangular APs case (Figure 3C), demonstrating the most reliable reconstruction in each case.

For all cases, the best activation map was obtained with 512-leads and a reliable solution with 256-lead with second order Tikhonov regularization.

\subsection{Reconstruction of re-entrant activation}

Different re-entry atrial activation patterns were also analyzed. Heterogeneous electrophysiology with remodelling was used.

Figure 4 shows snapshots of the reconstructed epicardial activation map of a re-entrant excitation with its activation centred on the sino-atrial node (SAN). Activation maps at $160,220,340$ and $500 \mathrm{~ms}$ after the initiation of the re-entry were reconstructed by using 512lead ECG and three Tikhonov regularization methods (Figure 4B-D), which were compared to the real activation map (Figure 4A). It was shown that the reconstruction with zero order Tikhonov regularisation method (Figure 4B) captured better the general feature of the re-entry as compared to the first and second order of the Tikhonov regularisation method (Figure 4C \& D). With the use of the zero-order Tikhonov regularisation method, though the reconstruction was not smooth, the key features of the re-entry, such as the location of the scroll-wave tip and gross shape of atrial activation were captured. However, the use of first and second order Tikhonov approaches did not produce a reliable reconstruction and failed to capture the spatially complex pattern (Figure 4C and D) due to the smoothing effects of the two methods. 


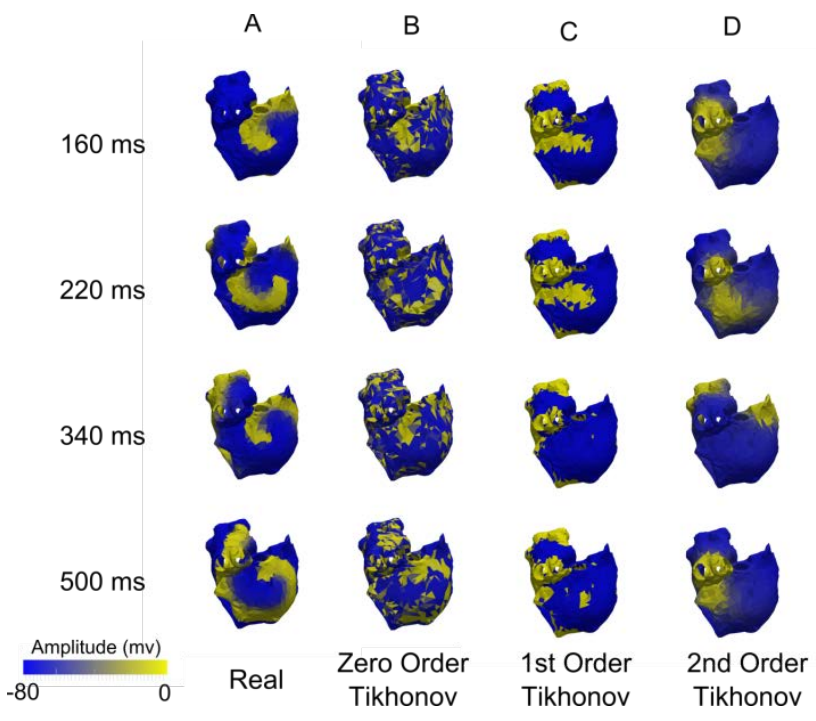

Figure 4. Snapshot at different timings (160, 220, 340 and $500 \mathrm{~ms}$ ) of reconstructed epicardial activation for a rotor wave with its origin located at the sino-atrial node region. (A) Real activation pattern; (B) Reconstructed pattern using zero oder; (C) Reconstructed pattern using first order; and (D) Reconstructed pattern using second order Tikohonov regularization method. All reconstructions were done by using 512-lead ECGs.

\section{Conclusion}

By using a biophysically detailed computer model of human atria-torso and different Tikhonov regularization methods we have reconstructed the epicardial atrial activation of arrhythmic excitation in association with both ectopic focal and re-entrant activities. Activation maps, isochrones and RE showed how the reliability of the different reconstruction methods depended on the morphology of the epicardial potentials and the rate of the activation.

\section{References}

[1] Rudy Y. Noninvasive Electrocardiographic Imaging of Arrhythmogenic Substrates in Humans. Circ Res. 2013 Jan 3;112(5):863-74.

[2] Jiang Y, Qian C, Hanna R, Farina D, Dössel O. Optimization of the electrode positions of multichannel ECG for the reconstruction of ischemic areas by solving the inverse electrocardiographic problem. Int $\mathrm{J}$ Bioelectromagn. 2009;11(1):27-37.

[3] Ghodrati A, Brooks DH, MacLeod RS. Methods of Solving Reduced Lead Systems for Inverse Electrocardiography. IEEE Trans Biomed Eng. 2007 Feb;54(2):339-43.

[4] Cuculich PS, Wang Y, Lindsay BD, Faddis MN, Schuessler RB, Damiano RJ, et al. Noninvasive Characterization of Epicardial Activation in Humans With Diverse Atrial Fibrillation Patterns. Circulation. 2010 Oct
5;122(14):1364-72.

[5] Dubois R, Shah AJ, Hocini M, Denis A, Derval N, Cochet $\mathrm{H}$, et al. Non-invasive cardiac mapping in clinical practice: Application to the ablation of cardiac arrhythmias. J Electrocardiol. 2015 Nov;48(6):966-74.

[6] Nattel S, Shiroshita-Takeshita A, Brundel BJ, Rivard L. Mechanisms of atrial fibrillation: lessons from animal models. Prog Cardiovasc Dis. 2005;48(1):9-28.

[7] Rahman F, Kwan GF, Benjamin EJ. Global epidemiology of atrial fibrillation. Nat Rev Cardiol. 2014;11(11):639-54.

[8] Colman MA, Aslanidi OV, Kharche S, Boyett MR, Garratt C, Hancox JC, et al. Pro-arrhythmogenic effects of atrial fibrillation-induced electrical remodelling: insights from the three-dimensional virtual human atria. J Physiol. 2013 Sep 1;591(Pt 17):4249-72.

[9] Narayan SM, Krummen DE, Rappel W-J. Clinical mapping approach to diagnose electrical rotors and focal impulse sources for human atrial fibrillation. J Cardiovasc Electrophysiol. 2012 May;23(5):447-54.

[10] J J, O B, A S, R M. Mechanisms of atrial fibrillation: mother rotors or multiple daughter wavelets, or both? J Cardiovasc Electrophysiol. 1998 Aug;9(8 Suppl):S2-12.

[11] Alday EAP, Colman MA, Langley P, Butters TD, Higham J, Workman AJ, et al. A New Algorithm to Diagnose Atrial Ectopic Origin from Multi Lead ECG Systems Insights from 3D Virtual Human Atria and Torso. PLoS Comput Biol. 2015 Jan 22;11(1).

[12] Colman MA, Aslanidi OV, Stott J, Holden AV, Zhang H. Correlation between P-wave morphology and origin of atrial focal tachycardia--insights from realistic models of the human atria and torso. IEEE Trans Biomed Eng. 2011 Oct;58(10):2952-5.

[13] Aslanidi OV, Colman MA, Stott J, Dobrzynski H, Boyett MR, Holden AV, et al. 3D virtual human atria: A computational platform for studying clinical atrial fibrillation. Prog Biophys Mol Biol. 2011;107:156-68.

[14] Kharche SR, Stary T, Colman MA, Biktasheva IV, Workman AJ, Rankin AC, et al. Effects of human atrial ionic remodelling by $\beta$-blocker therapy on mechanisms of atrial fibrillation: a computer simulation. Eur Eur Pacing Arrhythm Card Electrophysiol J Work Groups Card Pacing Arrhythm Card Cell Electrophysiol Eur Soc Cardiol. 2014 Oct;16(10):1524-33.

[15] Stenroos M, Haueisen J. Boundary element computations in the forward and inverse problems of electrocardiography: comparison of collocation and Galerkin weightings. IEEE Trans Biomed Eng. 2008 Sep;55(9):2124-33.

[16] Tikhonov A. Solutions of ill-posed problems.

[17] Hansen PC, O'Leary DP. The use of the L-curve in the regularization of discrete ill-posed problems. SIAM J Sci Comput. 1993;14(6):1487-503.

\section{Acknowledgements}

This work was supported by CONACyT and EPSRC project grant.

Address for correspondence.

Name. Erick Andres Perez Alday

E-mail address.

erickandres.perezalday@postgrad.manchester.ac.uk 